ausgewiesene Experten zu gewinnen. So porträtiert Eckhard Jesse DKP und NPD, Kai Oliver Thielking mehrere christliche Parteien und Uwe Kranenpohl die ödp. Die - durchaus kurzweilige - Lektüre zu den Klein- und Kleinstparteien mutet zuweilen wie der Gang durch ein Kuriositätenkabinett an. Einerseits liest man vergnügt die Berichte über die Spaßparteien, die mit abstrusen Forderungen offenkundig zur allgemeinen Erheiterung beitragen wollen. Andererseits stößt man auf extremistische Parteien, die sich offen oder verdeckt gegen die bundesrepublikanische Grundordnung stellen, und denen es vereinzelt sogar gelingt, ihre Mitglieder in eine sektenartige Abhängigkeit zu versetzen und finanziell sowie personell auszubeuten.

Die verschiedenen Porträts veranschaulichen, dass eine breite parlamentarische Präsenz für den Fortbestand einiger Parteien nicht zwingend notwendig ist. So waren oder sind etwa die Grauen und die ödp trotz begrenzter Wahlerfolge dauerhaft aktiv. Ferner verfestigt sich bei der Lektüre der von den Herausgebern beschriebene Eindruck (S. 10 f.), wonach vielfache Querverbindungen zwischen den behandelten Parteien bestehen. Umgruppierungen, Neugründungen, Umbenennungen, Fusionen und Abspaltungen haben in den letzten Jahrzehnten offenbar die Wirklichkeit vieler Klein- und Kleinstparteien bestimmt.

Das Nebeneinander der diversen Parteien verdeutlicht, wie unterschiedlich diese hinsichtlich ihrer politischen Erfolge und elektoralen Stabilität sind. So ist kaum absehbar, welche Parteien dauerhaft Bestand haben und welche dereinst als historische Pittoreske gelten werden. Dies unterstreicht nochmals die wissenschaftliche Relevanz der Faktoren und Momente, die Wahlerfolge ermöglichen und den Parteien die Perspektive zur Etablierung im politischen System eröffnen. Den Herausgebern und Autoren ist mit dem Handbuch ein kundiger, informativer und anregender Überblick gelungen, wobei sich die detaillierten Darstellungen der einzelnen Parteien und die einleitenden Aufsätze, die den allgemeinen Hintergrund beleuchten, sinnvoll ergänzen.

Heiko Biehl

\title{
Parteien in Parlament und Regierung: ein Beitrag zur Vergleichenden Regierungslehre
}

Saalfeld, Thomas: Parteien und Wahlen, Nomos Verlagsgesellschaft, Baden-Baden 2007, 350 Seiten, $€ 21,90$.

Politische Parteien umfassend darzustellen und zu analysieren ist kein leichtes Unterfangen: Sie sind sehr komplexe Gebilde mit vielschichtigen Organisationsstrukturen, nehmen eine Fülle von Funktionen wahr, die sie in einzelnen gesellschaftlichen und politischen Bereichen erfüllen sollen. Gleichzeitig agieren sie in Mehrebenensystemen auf lokaler, regionaler, nationaler und auch supranationaler Ebene. In parlamentarischen Demokratien gelten sie nach wie vor als unersetzliche Akteure im politischen Willensbildungsprozess, organisieren und koordinieren gesellschaftliche, parlamentarische und gouvernementale Prozesse mit.

Thomas Saalfeld unternimmt in diesem als Studienkurs konzipierten Buch den Versuch einer umfassenden Darstellung politischer Parteien, konzentriert sich dabei aber auf die Vorstellung von Ergebnissen aus hauptsächlich drei Bereichen der Forschung: Parteien in der Wählerschaft, Parteien als außerparlamentarische Organisationen und als Akteure in Parlamenten und Regierungen. Letzterer Aspekt wird dabei überraschend ausführlich be- 
handelt, obwohl der Titel des Bandes eher die elektorale Seite des Parteihandelns hervorhebt. So erfährt der Leser entsprechend viel Wissenswertes über die Rolle der Parteien zur Herstellung von Regierungsmehrheiten, zur Herbeiführung von Regierungsstabilität oder zur Kontrolle des administrativen Regierungsapparates wie auch über ihr Wirken in Parlamenten, beispielsweise aber wenig über die Rolle von Parteien als Wahlkampforganisationen, als Organisationen zur Rekrutierung des politischen Personals oder über ihre Funktionen der Mobilisierung der Bürger und der Interessenaggregation, also letztlich über die Wählerschaften von Parteien. Diese Selektion ist wohl mit Saalfelds Perspektive zu erklären: Er sieht politische Parteien einerseits primär aus der Sicht des Rational Choice-Ansatzes, andererseits immer mit Blick auf ihr Wirken und ihre Handlungsoptionen in den Institutionen oder in dem durch die Verfassungsinstitutionen vorgegebenen Rahmen eines politischen Systems. Gemäß dieser Sichtweise liegt es näher, etwa Ergebnisse der Forschung zu Wahlsystemen, Koalitionstheorien oder zur parlamentarischen Agenda-Setzer-Macht darzulegen als zu normativen Aspekten wie Funktionen von politischen Parteien, zu sozialen Fragestellungen des Wandels der Wählerstrukturen mit ihren Auswirkungen auf Parteiendemokratien oder zu kommunikativen Aspekten wie Parteien in Wahlkämpfen. Aspekte der Parteiorganisation tauchen demnach allenfalls am Rande auf.

Auf eine bewusste Verengung seiner Perspektive weist Saalfeld selbst hin, indem er seinen - wie er selbst einräumt - historisch und strukturell verkürzten Parteibegriff in der Einleitung definiert, wonach „Parteien von Politikern geschaffene Organisationen sind, die den Machtgewinn und der Erreichung ihrer eigenen sachpolitischen Ziele dienen“" (S. 14 f.). Auf dieser Grundlage entwickelt der Autor eine primär institutionelle Sichtweise auf Parteien, deren Handeln in den konsolidierten west-, nord- und südeuropäischen Demokratien er auf der Basis einer akribischen Auswertung der Ergebnisse politikwissenschaftlicher Forschung und mit Aufbereitung sehr zahlreicher empirischer Aggregatdaten in zwölf Kapiteln genauestens analysiert. Es gelingt ihm, Verbindungslinien zwischen den verschiedenen Forschungsgebieten zu ziehen, somit die unterschiedlichen Handlungsfelder von Parteien in einem Gesamtkontext darzustellen und dem Leser vor Augen zu führen. Er wird damit seinem Anspruch gerecht, die Wechselwirkungen von Verfassungsinstitutionen, Strukturen des Parteiensystems und Strategien der Parteien in den Mittelpunkt zu stellen. In diesem Zusammenhang muss jedoch erwähnt werden, dass der strategische Aspekt sich hauptsächlich wieder auf das Handeln innerhalb der Verfassungsinstitutionen richtet und weniger auf das Gewinnen von Wahlen, die strategische Ausrichtung von Wahlkämpfen oder die (wahl-)strategische Positionierung im Parteiensystem. Ausführlich behandelt werden entsprechend Wahlsysteme mit ihren Auswirkungen auf die Strukturen von Parteiensystemen und Parteien als Akteure in Parlamenten und Regierungen. Hier reflektiert Saalfeld theoretische Modelle und Konzepte wie empirische Ergebnisse und informiert den Leser umfassend etwa über Regierungsstabilität im internationalen Vergleich wie über parlamentarische Interpellations- und Fragerechte. Zahlreichen Abbildungen und Tabellen sind detaillierte Informationen zu entnehmen, die Saalfeld auch in den Gesamtkontext einordnet und interpretiert. Doch nur in zwei Kapiteln kommen zentrale Felder der Parteienforschung wie Parteiorganisationen, Typologien, programmatisch-ideologische Positionen und Differenzen von Parteien, Mitgliederentwicklung, Parteifinanzen, Aspekte innerparteilicher Demokratie oder die Eigenschaften von Parteiensystemen vor. Für ein Lehrbuch über Parteien wäre es wünschenswert gewesen, wenn dieser Teil des Buches deutlich mehr Gewicht bekommen und der Autor anschaulicher formuliert hätte. Für ein Lehrbuch erscheint 
die überwiegend an jüngeren Forschungsergebnissen orientierte Darstellung auch zu detailliert. Feinheiten der vielen dargebotenen Modelle und theoretischen Perspektiven wie Konzeptionen erscheinen verzichtbar.

Saalfelds Buch ist somit letztlich weder als klassisches Lehrbuch über politische Parteien zu verstehen noch als eine umfassende Darstellung zu Parteien auf der elektoralen Ebene. Es bietet stattdessen einen informativen, umfassenden und fundierten Überblick der vergleichenden Forschung zu westlichen Regierungssystemen mit Schwerpunktsetzung auf den unterschiedlichen Formen des Handelns von Parteien und deren Wirken in Regierung und Parlament. Wer in diesem Feld der Vergleichenden Regierungslehre nach einer Einordnung und Interpretation von theoretischen Modellen und empirischem Wissen sucht, der sollte den Band zur Hand nehmen.

Uwe Jun

\section{Parteibeitritt und seine Motive: wenig Neues aus der Forschung}

\section{Biehl, Heiko: Parteimitglieder im Wandel. Partizipation und Repräsentation, VS Verlag für Sozialwissenschaften, Wiesbaden 2005, 265 Seiten, € 39,90.}

In dem aus seiner Dissertation hervorgegangenen Buch befasst sich Heiko Biehl, wie der Untertitel ankündigt, mit den Motiven für einen Parteibeitritt (Partizipation) und mit dem Grad der Übereinstimmung von politischen Orientierungen bei Parteimitgliedern und Parteianhängern (Repräsentation). Beide Problembereiche werden mittels der bekannten These vom „neuen Typ“ von Parteimitgliedern verklammert. Sie behauptet folgende Wandlungstendenzen: Parteien würden ihre Mitglieder immer häufiger aus denselben, ressourcenstarken sozialen Gruppen rekrutieren und sich folglich hinsichtlich der Sozialstruktur ihrer Mitglieder weiter angleichen. Gleichzeitig veränderten sich auch die Beitritts- beziehungsweise Bindungsmotive von eher gesinnungs- und gefühlsmäßigen zu eher instrumentellen Aspekten. Die Repräsentationsproblematik gelangt dadurch ins Spiel, dass der Verfasser aus dem sozialstrukturellen Angleichungsprozess die Frage ableitet, ob die Mitglieder aller Parteien womöglich „gleiche oder zumindest ähnliche politische Überzeugungen“ oder gar „ihre eigenen Interessen“ (S. 183 f.) vertreten, womit die Parteien ihrer Funktion als intermediäre Institutionen nicht gerecht würden.

Die Annahme, dass Personen in ähnlicher sozialer Lage übereinstimmende politische Orientierungen entwickeln, erscheint dem Rezensenten allerdings wenig wahrscheinlich. Verdankt sich die Entstehung der empirischen Einstellungsforschung doch letztendlich der Einsicht, dass das Sein eben nicht das Bewusstsein bestimmt. Die empirischen Analysen führen dann auch zu dem Ergebnis, dass die Parteien diejenigen Standpunkte vertreten, „die auch in der Bevölkerung bzw. in der jeweiligen Anhängerschaft Anklang finden“ (S. 198). Jedenfalls lautet die Leitfrage der Untersuchung, ob sich ein Wandel der Parteimitglieder hinsichtlich ihrer Sozialstruktur und ihrer Beitrittsmotive vollzogen hat und ob diesbezüglich Ost-West-Unterschiede zu beobachten sind.

Die These vom „neuen Parteimitglied“ wird erstmalig auf einer außerordentlich breiten Datengrundlage untersucht. Sie besteht aus einer Befragung von Mitgliedern aller Bundestagsparteien sowie einer Bevölkerungsumfrage, die zeitgleich im Frühjahr 1998 durchgeführt wurden. Diese „Potsdamer Parteimitgliederstudie“ war bereits für 2003 im Westdeutschen 